\title{
Progress in the production of the W7-X divertor target modules
}

\author{
J. Boscary ${ }^{\mathrm{a}}$, G. Ehrke ${ }^{\mathrm{b}}, \mathrm{H}$. Greuner ${ }^{\mathrm{a}}$, P. Junghanns ${ }^{\mathrm{a}}, \mathrm{C} . \mathrm{Li}^{\mathrm{a}}$, \\ B. Mendelevitch ${ }^{\mathrm{a}}$, J. Springer ${ }^{\mathrm{a}}$, R. Stadler ${ }^{\mathrm{a}}$, and the W7-X Team \\ ${ }^{a}$ Max-Planck-Institut für Plasmaphysik, Boltzmannstr. 2, 85748 Garching, Germany \\ ${ }^{b}$ Max-Planck-Institut für Plasmaphysik, Wendelsteinstr. 1, 17498 Greifswald, Germany
}

\begin{abstract}
The realization of the $19.6 \mathrm{~m}^{2}$ highly heat loaded target area of the actively water-cooled divertor of Wendelstein 7-X (W7-X) requires the installation of 100 target modules distributed in ten discrete similar divertor units. A target module is water-cooled from manifolds to distribute the water equally between the target elements with flanged connections to the water supply system. A target element is made of a $\mathrm{CuCr} 1 \mathrm{Zr}$ copper alloy heat sink armored with CFC NB31 tiles and designed to remove a stationary heat flux up to $10 \mathrm{MW} / \mathrm{m}^{2}$. The production of the first 30 target modules of the high iota tail has been successfully completed in the workshop of IPP-Garching. The quality of the finished target modules was systematically assessed as follows: visual inspections, measurement of the 3-D CFC target area, dynamic pressure tests, integral He leak testing under pressure at different conditions $\left(20^{\circ} \mathrm{C}, 4 \mathrm{MPa}\right.$ and $160^{\circ} \mathrm{C}, 2.5 \mathrm{MPa}$ ) in a vacuum oven, high heat flux testing. Only 2 finished target modules did not pass the integral He leak testing and were successfully repaired.
\end{abstract}

Keywords: Stellarator, Wendelstein 7-X, Plasma facing component, Divertor.

\section{Introduction}

After coming into operation with a limiter [1], the stellarator Wendelstein 7-X (W7-X) started short pulse operation with an adiabatically loaded divertor made of fine-grained graphite tiles [2]. The next step is the machine upgrade for operation with pulse length up to $1800 \mathrm{sec}$. and continuous plasma heating with up to 10 MW electron cyclotron resonance heating (ECRH). For long pulse operation, an actively water-cooled HHF (high heat flux) divertor together with a cryo-vacuum pump system [3] needs to be installed. The function of the divertor is the controlled heat and particle exhaust from the plasma. The W7-X divertor consists of 10 similar discrete divertor units, two units per period. The design of the divertor surface is based on nine reference plasma configurations. Its extensive total area of $25.6 \mathrm{~m}^{2}$ offers a high degree of experimental flexibility [4] and consists of four main areas: the vertical area positioned on the inboard side of the plasma vessel, the horizontal area positioned on the outboard side of the plasma vessel with an intermediate lower loaded surface (Fig. 1). The whole divertor surface was originally designed to remove stationary heat fluxes of $10 \mathrm{MW} / \mathrm{m}^{2}$. The design was modified to reduce the highly loaded surface without reducing physics objectives. The lower loaded area has been designed for stationary heat fluxes up to $1 \mathrm{MW} / \mathrm{m}^{2}$ and has a total surface of $6 \mathrm{~m}^{2}$ [5]. This paper focuses on the progress in the production of the target modules (TMs) of the area with a toroidal angle between $-12^{\circ}$ and $-28^{\circ}$ (Fig.1). In a first part, the preparation phase of the production is presented. The second part describes the manufacturing process of the TMs. Finally, the result of the quality assessment of the production is presented.

\section{Preparation phase}

A call for tender for the production in industry of the TMs was not successful, therefore the production was split into two parts: the production of the target elements
(TEs) awarded to industry (Plansee SE, Austria), the manufacturing of the TMs in the Integrated Technical Centre (ITZ) of IPP-Garching.

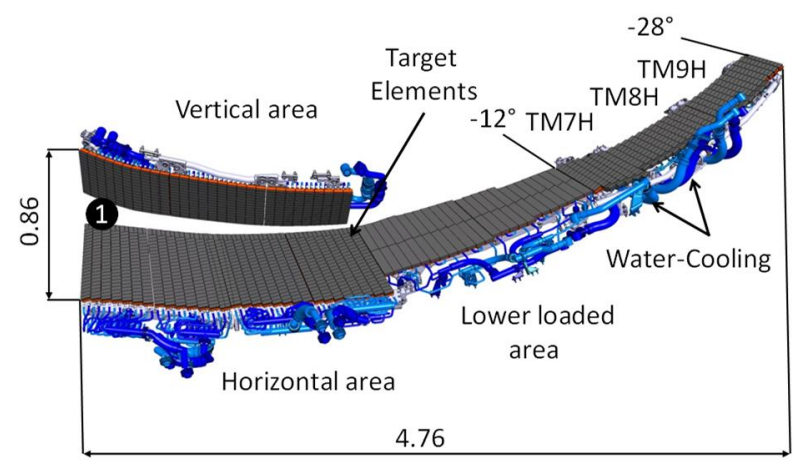

Fig. 1. Overview of one divertor unit (TM: target module). Outer dimensions are in $\mathrm{m}$. The divertor is an open structure with the horizontal and vertical areas, which define the main pumping gap (1). Other divertor components such as the baffles and divertor closures are not shown.

\subsection{Target elements}

Basically, the highly loaded components, namely the TEs, define the divertor performance. A TE is made of a $\mathrm{CuCr} 1 \mathrm{Zr}$ copper alloy heat sink armored with carbon fibre reinforced carbon $\mathrm{CFC} \mathrm{NB} 31$ tiles and designed to remove a stationary heat flux of $10 \mathrm{MW} / \mathrm{m}^{2}$. Many efforts with an extensive testing programme were needed to achieve the required performance [6]. Performance indicators of TE production are given in [7]. About $8 \%$ of the TEs were HHF tested in the GLADIS [8] facility at IPP. The performed statistical assessment confirmed the stable production and the high thermal performance of the delivered TEs [9].

\subsection{Target modules}

The production and quality control of the TMs in ITZGarching had the advantage to involve an experienced team in the design and manufacture of components for experimental fusion machines such as ASDEX Upgrade. 
In retrospect this decision allowed the fast and efficient interaction between the workshop and the design office as well as the required flexibility to find solutions during the production on site and to take late design change into account.

The divertor operation would be restricted if the built divertor surface does not remain within the specified tolerances. Therefore, before launching the production, a TM prototype using TE prototypes was manufactured and finally tested in the GLADIS facility. The main results were: The design of the support structure and of the attachment system of the TEs to this structure allows the realization of the divertor surface of a TM within the specified tolerances, (2) The required surface tolerance can be achieved with the surface machining of each individual TE instead of a the whole surface of a finished TM, (3) The analysis of the experimental results together with calculations using finite element method confirmed that the TM could withstand the specified operating loads of W7-X, regarding stress and strain distributions. All details can be found in [5].

\section{Manufacturing of the target modules}

The manufacturing started with the horizontal TM9H, then $8 \mathrm{H}$, and finally $7 \mathrm{H}$ (Fig. 1) to be in phase with the delivery sequence of the TEs. For operation in W7-X, stainless steel is specified with a cobalt content $\leq 500$ ppm, and a relative magnetic permeability $\leq 1.01$, except weld seams. The material charges were well identified with certificates for traceability. Own measurements of the material composition and permeability were always performed and no deviations were detected by IPP.

\subsection{Surface of the target module}

Fig. 2 shows a finished TM9H installed onto its frame for storage and transport. The CFC surface is made of consecutive TEs in the toroidal direction which were individually 3D machined [10]. Each TE is identified with a serial number engraved on its back side and controlled before welding. The TEs were manufactured with two $\mathrm{CuCr} 1 \mathrm{Zr}$ studs and screwed onto the rails (Fig. 3) via disc spring stacks with a $7 \mathrm{Nm}$ torque [11]. The rails were joined by a stiffening support plate made of stainless steel forming a frame. The bearing surfaces, which defines the position of the TEs, were very precisely machined (surface tolerance between two neighboring surfaces of $0.05 \mathrm{~mm}$ ) after the assembly of the frame. The rails were manufactured within the tolerances and no rework was necessary. In one rail, holes were machined for fixed points and in the other rail slotted holes for thermal expansion in the length direction. This solution should allow neighboring elements moving together to avoid leading edge in operation.

The 3D-machined TEs were firstly positioned onto the frame before welding to the cooling pipes. The steps between neighboring TEs within a TM (requirement: $\pm 0.1 \mathrm{~mm}$ ) were systematically measured with a Zeiss machine of type Accura II. Each point of the 3D-surface was compared to the corresponding points of the CADsurface (requirement: $\pm 0.5 \mathrm{~mm}$ ) in the same coordinate system. This procedure was systematically repeated at the end of the manufacturing process to confirm that the subsequent manufacturing steps did not alter the area. Thanks to this approach, no deviations occurred. No bearing surfaces of the supporting structure needed to be reworked. The produced TMs met all these requirements.

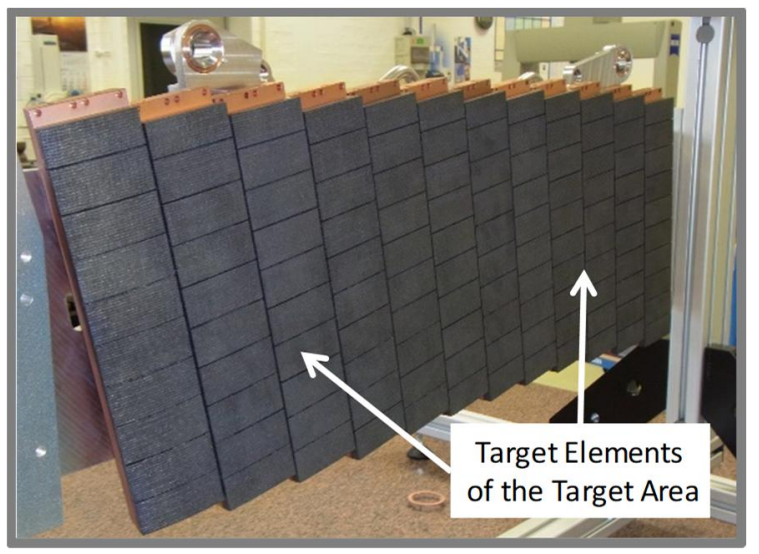

Fig. 2. Finished TM9H: plasma-facing surface. $0.8 \mathrm{~mm}$ slits between CFC NB31 tiles are visible.

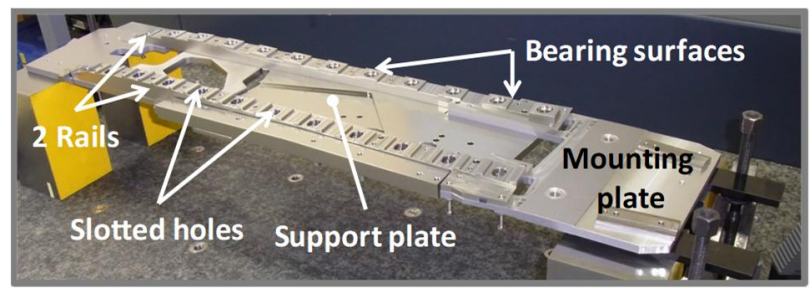

Fig. 3. TM frame for supporting TE

\subsection{Cooling system}

The complicated and compact piping arrangement of the TMs (Fig. 4) is due to the 3D shape of the plasma vessel and the restricted available space. The completed piping system is the connection of two sub-groups: manifolds + pipes, and TEs + pipes. For each connection, a qualification phase following the W7-X standards was performed to set up the parameters of the welding programme. The pipe geometry is $\varnothing 12 \mathrm{~mm}, 1 \mathrm{~mm}$ thick. Material is X2CrNiMo17-13-3 according to DIN 1.4429. At the very beginning of the design, TIG (Tungsten Inert Gas) orbital welding with full penetration and without additional material was selected for the butt pipe welding. The consequence of the systematic CAD collision check was the design of segmented support frames to allow the pipe integration.

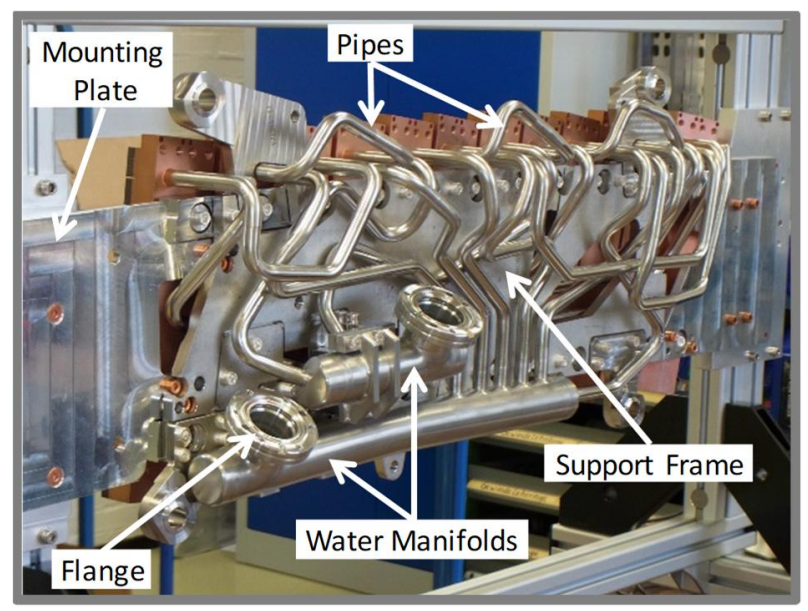


Fig. 4. Finished TM9H: Backside and water-cooling system. Flanges allow the connection to the water-cooling supply.

The manifolds were procured from the company Dockweiler, Germany, which developed the "collaring" technology. This solution allows inside welding of branch stubs to avoid gaps. This technology was selected because it was already successfully used for the production of hundreds of manifolds for the water-cooling system of the W7-X first wall components [12]. The manifolds $(\varnothing 38 \mathrm{~mm}, 2 \mathrm{~mm}$ thick) were delivered with branches, $17.5 \mathrm{~mm}$ long, and with CF-flanges to connect to the water supply system. For the production of the 60 manifolds, 8 NCRs (Non-Conformity Reports) were released: 6 for a slightly higher relative permeability of 1.08 in the weld seam than the specified value of $1.05,1$ for the sealing lips of the CF flange slightly damaged (46 manifolds) during inspections which required slight additional machining to remove scratches, 1 for one branch of 10 manifolds of the same type, which had a 5 $\mathrm{mm}$ shorter branch than specified. The length of the additional pipe to be welded to the branches depended on the positioning for final assembly to avoid collision with other pipes and the support frame, and on the position of the last weld to close the circuit (Fig. 5). Many special jigs were manufactured to set precisely the 3D positions and axes of the ends. Special attention was given to the preparation and positioning of the chamfered pipe ends to get parallel surfaces without gap.

At the beginning of the production, unexpected difficulties appeared for the welding of the TE connectors to the pipes. The connector is the transition pipe between $\mathrm{CuCr} 1 \mathrm{Zr}$ and stainless steel pipes made of X2CrNiMo1712-2 according to DIN 1.4404 with a Ni adapter. It was produced from blocks which were electron beam welded and then machined to the pipe dimension. The examination according to DIN EN ISO 5817 of all first weld seams showed imperfections corresponding to an incompletely filled groove $\leq 0.2 \mathrm{~mm}$ on one side of the seam (i.e. the seam thickness was not constant), also confirmed with X-ray inspections and metallographic analyses.

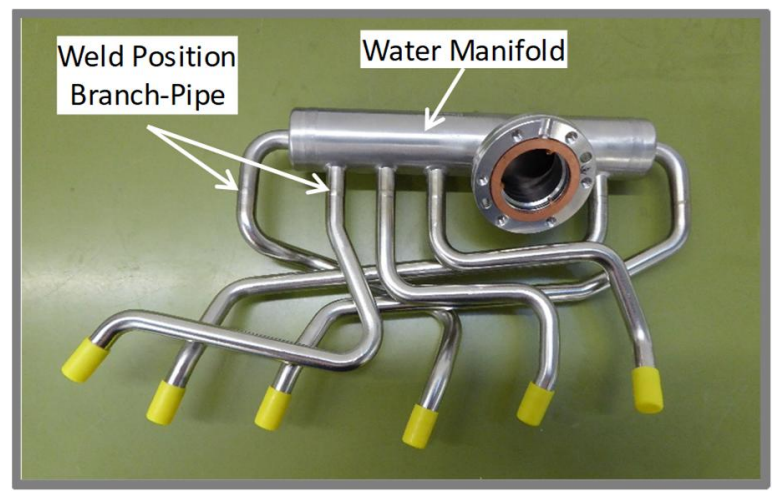

Fig. 5. Example of one manifold with welded pipes the for TM7H before assembly in the TM. A Cu-ring protects the lip of the CF-flange.

After many inquiries to understand this phenomenon, the explanation was found finally in the chemical composition. 1.4404 has a specified sulfur content of $0.030 \%$ and 1.4429 of $0.015 \%$, respectively. This slight difference produced the deviation of the beam towards the side of higher sulfur content, the so-called Marangoni effect [13]. Many additional test pieces were welded and analyzed: the metallographic analyses showed no cracks and porosities, He leak test performed (inside the test pieces evacuated) were successful (leak rate $\leq 10^{-7} \mathrm{~Pa} / \mathrm{s}$ at room temperature). It was demonstrated that this weld seam was reliable, and the process was released.

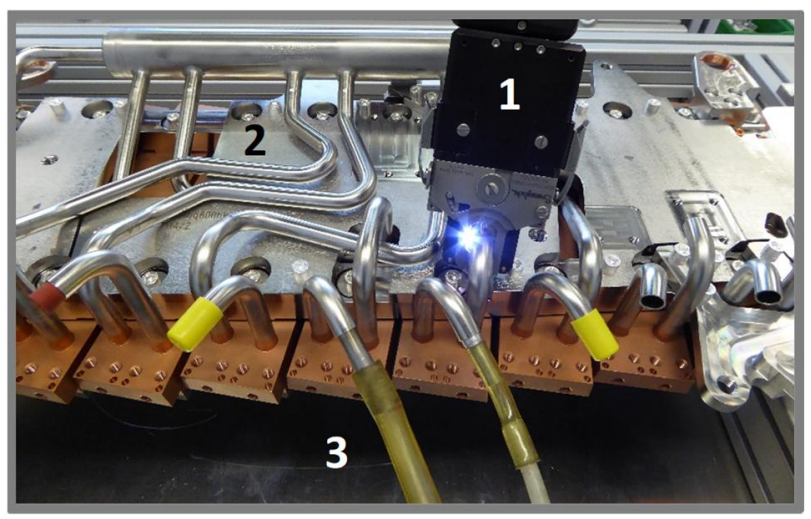

Fig. 6. Example of orbital welding for TM8H. (1): positioned welding head, (2): TM support frame, (3) Ar supply to prevent heat from tinting. The welding is performed without extra wire (1 mm thick tube). Only, the first manifold was positioned at this stage.

For all connections, the pipes were spot welded and the positions were again checked before welding to avoid possible collisions. Fig. 6 shows the orbital welding between TEs and manifold pipes. During welding, the pipes were filled with Ar; the rest oxygen content in the pipe was always monitored and kept $\leq 20 \mathrm{ppm}$. No discoloration was allowed. The welds were systematically controlled by a visual inspection from outside and inside with an endoscope, when possible. Fig. 6 also shows the short pipes already welded to the water connectors of the TEs.

For TM9H and TM8H, TEs were connected two by two in series. The final stage was the weld between the manifolds and the TEs. The piping arrangement (Fig. 5) has the advantage to accommodate stresses during mounting and compensate for the movement of TEs under thermal loading by the flexibility of the pipes. A total of 1620 orbital welds was needed to complete the cooling system.

The W7-X water cooling system has to provide the same cooling conditions for each TE in each TM. Due to the parallel cooling arrangement of the TEs, only TEs with the same pressure drop were arranged in TM to get the same pressure drop in a given module. This selection was necessary because the measured pressure drops for a given type of TE had a $\pm 15 \%$ tolerance with respect to the measured mean value. In TM9H and TM8H, each branch has two TEs in series: 12 TEs and 6 branches. Calculations performed with the FloMASTER Software were in good agreement with measurements $(\leq 10 \%)$. The specified cooling conditions are: maximal inlet temperature $33^{\circ} \mathrm{C}$; static pressure: $1 \mathrm{MPa}$. The working point is an axial velocity of $10 \mathrm{~m} / \mathrm{s}( \pm 1 \mathrm{~m} / \mathrm{s})$ in each TE with a maximal temperature increase of $50 \mathrm{~K}$. The dynamic pressure drop of the TMs was systematically measured. At the working point, the results are: $0.9 \mathrm{MPa}$ 
for TM7H, 1.4 MPa for TM8H and 1.5 MPa for TM9H. These values met the specification. The scattering between the TMs of a same type was $\leq 8 \%$. The flow distribution between the TEs was also measured and calculated with FloMASTER. Both showed a maximal difference of $3 \%$ in the flow distribution within TMs.

\subsection{Results of the quality assessment}

Main features of the TMs are shown in Table 1.

Table 1. Main features of TMs

\begin{tabular}{lcccc}
\hline $\begin{array}{l}\text { TM } \\
\text { type }\end{array}$ & $\begin{array}{c}\text { Length } \\
(\mathrm{mm})\end{array}$ & $\begin{array}{c}\text { Width } \\
(\mathrm{mm})\end{array}$ & $\begin{array}{c}\text { Height } \\
(\mathrm{mm})\end{array}$ & $\begin{array}{c}\text { Weight } \\
(\mathrm{kg})\end{array}$ \\
\hline TM7H & 480 & 400 & 195 & 32 \\
TM8H & 760 & 352 & 155 & 55 \\
TM9H & 720 & 440 & 160 & 55 \\
\hline
\end{tabular}

The final stage of the quality assessment is the thermal load testing, the He leak rate and outgassing inspection, and the visual inspection before packaging.

The GLADIS facility played a key role in the development phase as well as in the quality assessment of the produced TMs. As for TEs, $10 \%$ of the TMs (1 TM per type) will be HHF tested up to the design load of 10 $\mathrm{MW} / \mathrm{m}^{2}$. The results confirmed the high required performances and stable manufacturing process: the attachment system and the cooling system withstood the specified HHF loading conditions. All tested TMs in GLADIS successfully passed the He leak rate inspection. The visual inspection showed no damage and deformation of the target area. The 3D measurement showed no change of the TM surface after HHF testing. In GLADIS, the heat flux distribution is well defined and the calibrated infrared camera has an optimal position to view the loaded area. The data of the spatial surface temperature distributions obtained at different heat flux levels can be used as reference for local heat flux calculations during plasma operation in $\mathrm{W} 7-\mathrm{X}$.

The He leak rate and outgassing were systematically monitored in a vacuum oven: a leak rate $\leq 510^{-7} \mathrm{Pal} / \mathrm{s}$ at room temperature and $4 \mathrm{MPa}$ internal He pressure, and a leak rate $\leq 510^{-6} \mathrm{~Pa} \ell / \mathrm{s}$ at $160^{\circ} \mathrm{C}$ and $2.5 \mathrm{MPa}$ internal $\mathrm{He}$ pressure $(1$ cycle). During the cycle, the mass spectrometer measurement was compared to a reference spectrum, which indicated the acceptable levels for W7$\mathrm{X}$. Only $2 \mathrm{TM} 7 \mathrm{H}$ did not pass the integral leak rate test, with three leaks over 1620 produced welds. The leak were detected by sniffing and all were localized at the weld seam between the TE connectors and manifold pipes. The successful repair process is published in [14].

\section{Conclusion}

The production of the $30 \mathrm{TMs}$ (TM7H, TM8H, TM9H) for the high iota tail of the HHF divertor has been successfully finished: 8 NCRs were issued for the manifolds and 2 NCRs for the finished TMs (He leak testing). No reserve TM was produced. The reduced number of NCRs validate the development activities carried out to prepare this production.

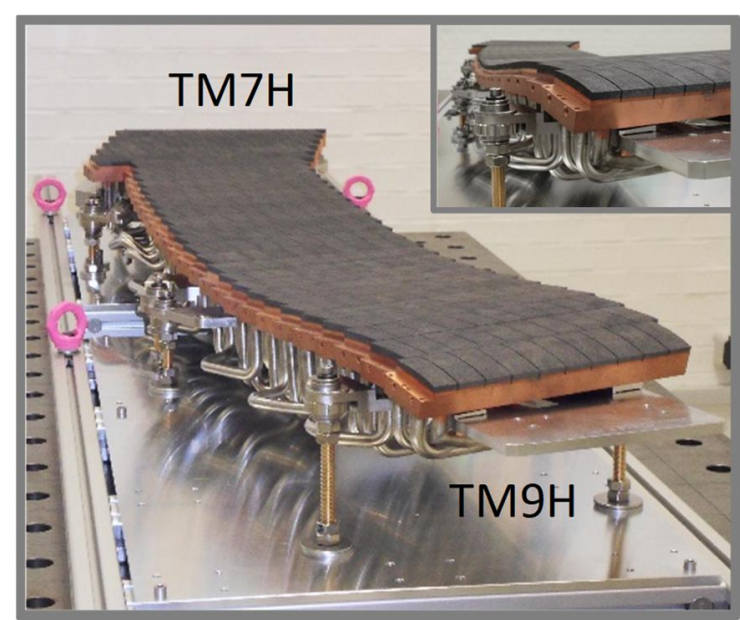

Fig. 7. Assembly trial of the target area of the high iota tail (foreground: TM9H).

Despite a complicated piping route and difficulties encountered during welding, the result of the quality assessment showed that all TMs met the specification: the TMs are tight and provide the required cooling conditions with an uniformly distributed flow between the TEs for long pulse operation with stationary heat flux of 10 $\mathrm{MW} / \mathrm{m}^{2}$. The very precise positioning of the TEs provides the required smooth 3D target area. In W7-X, the loading divertor surface conditions depends on the selected scenario. The challenge was to guarantee the same high performance on the whole divertor surface. The production of the remaining TMs will be completed on time.

\section{References}

[1] T. S. Pedersen, et al., Phys. Plasmas 24 (2017) 055503.

[2] A. Peacock, et al., Fusion Eng. Des. 84 (2009) 1475-1478.

[3] G. Ehrke, et al., Design and Manufacturing of the Wendelstein 7-X Cryo-Vacuum Pump, This conference.

[4] H. Renner, et al., Fusion Sci. Technol. 46 (2004) 318-326.

[5] A. Peacock et al., IEEE Trans. Plasma Sci. 42(3) (2014) 524-532.

[6] J. Boscary, et al., Fusion Eng. Des. 84 (2009) 497-500.

[7] J. Boscary, et al., Fusion Eng. Des. 124 (2017) 348-351.

[8] H. Greuner et al., J. Nucl. Mat. 367-370 (2007) 1444.

[9] H. Greuner, et al., Fusion Eng. Des. 88 (2013) 581-584.

[10] P. Junghanns, et al., Fusion Eng. Des. 98-99 (2015) 12261230.

[11] M. Smirnow, et al., Fusion Eng. Des. 89 (2014) $1037-$ 1041.

[12] B. Mendelevitch, et al., Fusion Eng. and Des. 88 (2013) 1660-1663.

[13] K. C. Mills, et al., Phil. Trans. R. Soc. Lond. A 356 (1998) 911-925.

[14] P. Junghanns, et al., Repair processes of W7-X target modules, This conference. 